\title{
Visualisation for ontology sense-making: A tree-map based algorithmic approach
}

\author{
Kaneeka Vidanage, Noor Maizura Mohamad Noor, Rosmayati Mohemad, Zuriana Abu Bakar
}

Faculty of Ocean Engineering Technology and Informatics, University Malaysia Terengganu-UMT, Kuala Terengganu, Terengganu, Malaysia

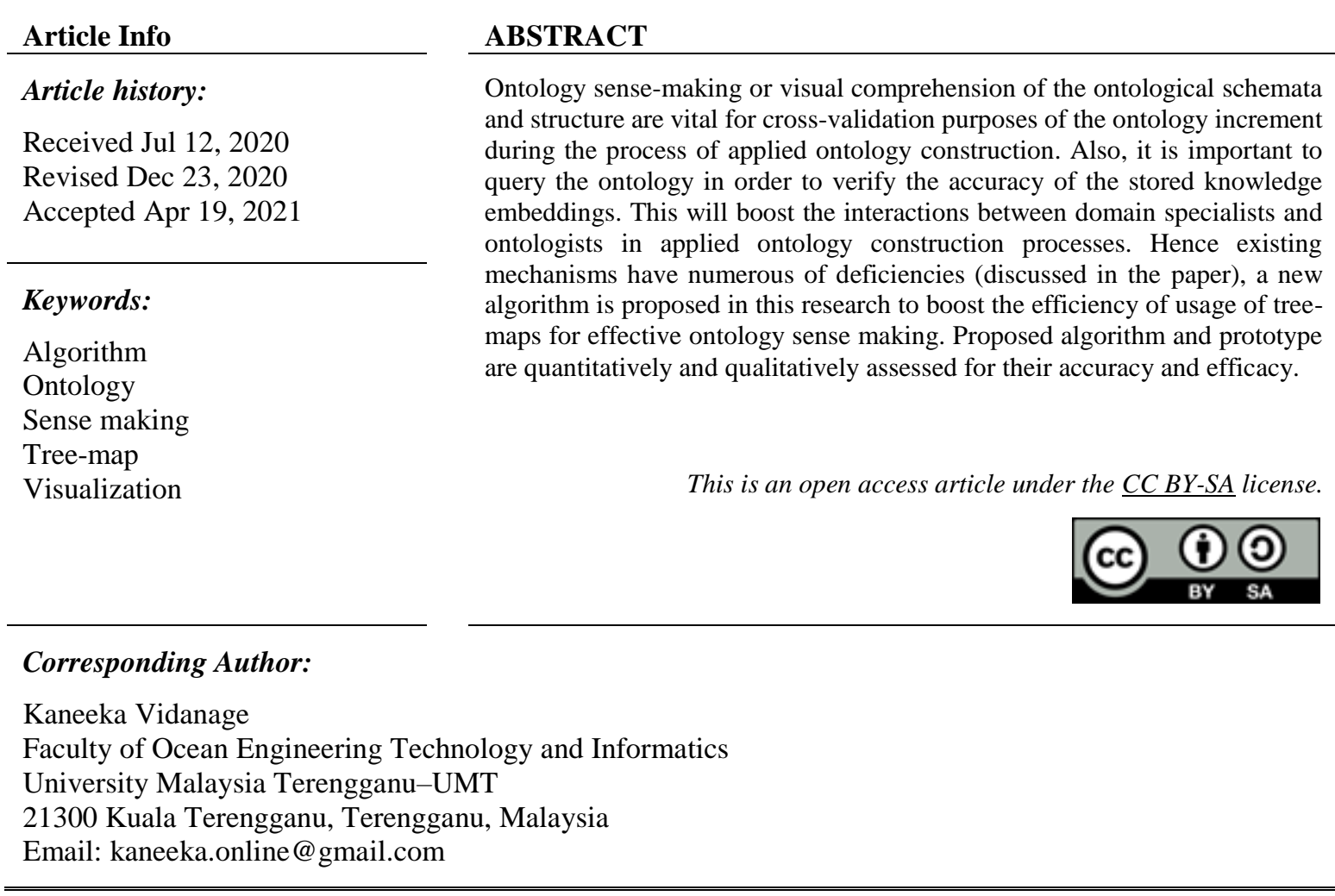

\section{INTRODUCTION}

In ontological taxonomy or structure assessment 'visualization compactness 'is an insolvable problem, since the screen size will become a fixed barrier [1], [2]. However, visualization clarity can be enhanced via rational blend of appropriate visualization techniques [3]. In applied ontology construction processes non-computing domain specialists also contribute for the knowledge modelling aspects. As claimed by [4], visualisation complexity is an adversely affecting bottleneck, which hinders their (i.e. domain specialists) active contribution to the process of applied ontology construction. [5] claim visualisation as a dire necessity for 'ontology sense making'. Hence, it is an active research niche, where researchers examine for better visualisation techniques to facilitate effective 'ontology sense making'. This paper discusses the existing challenges associated with ontology visualisation and proposes a resolution to overcome existing challenges. As elaborated in the related work section beneath, among multiple visualization techniques, tree-maps are commended for their drill down enabled traversal facility and knowledge abstraction aspects contributing for the simplicity [15], [16]. Hence, the main objective of this research is to propose a fully automated tree-map generation algorithm, which can function regardless of the domain or the schemata to facilitate ontology sense making. The proposed solution is evaluated by both domain specialists and ontologists, with the intension of providing a refined reflection. 


\section{RELATED WORK}

\subsection{Challenges}

\subsubsection{Scale vs. amount of information to be displayed}

Displaying all significant information, without mentally overloading the stakeholder is still an open challenge [6]. As a result of increased information density, visual clutter and occlusion occur. This makes the ontology sense making a complex task. Refer Figure 1 for more details.

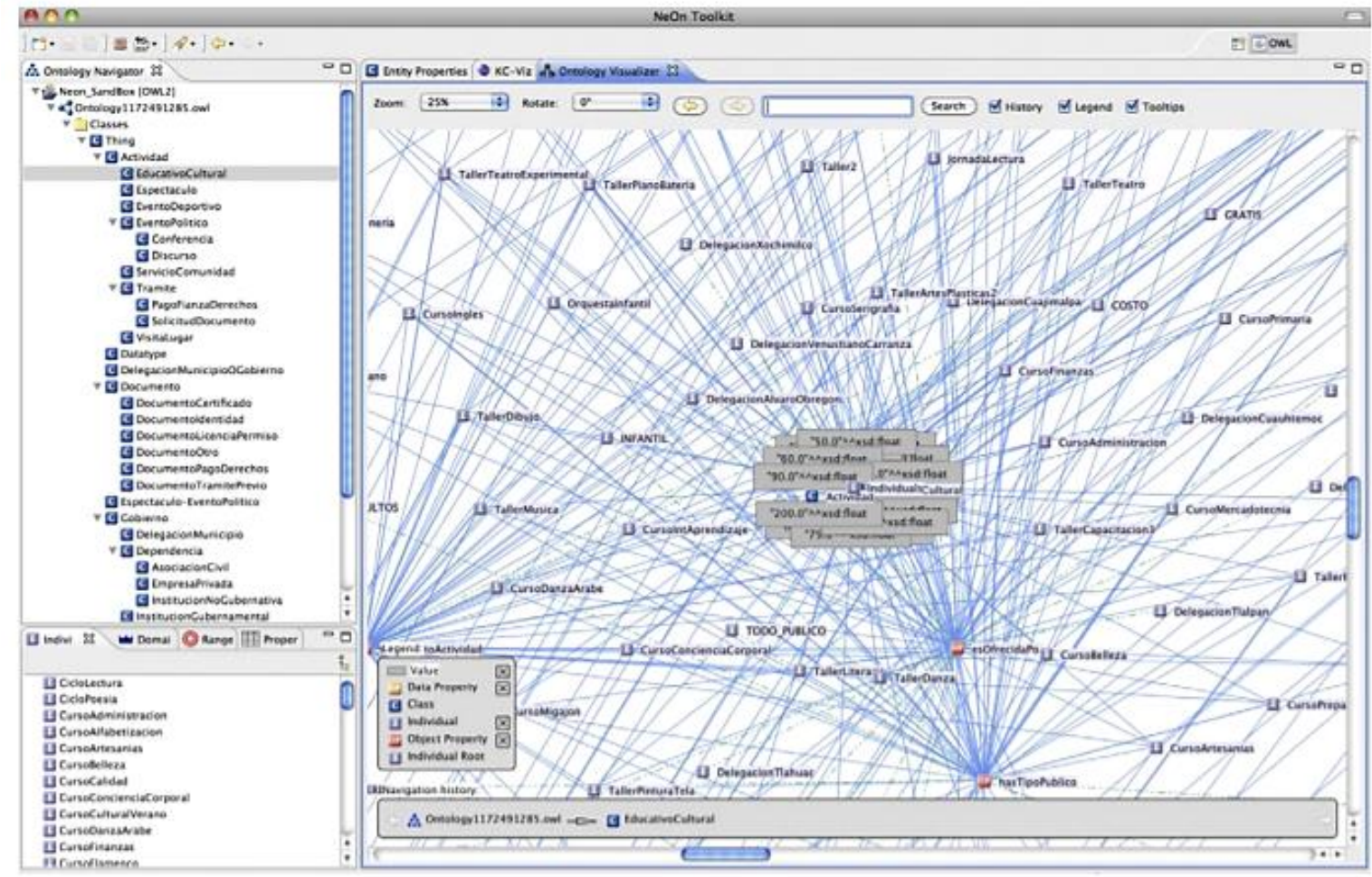

Figure 1. Occlusion and clutter in visual canvas

\subsubsection{Cognitive complexity}

When displaying a complex ontology schema, presentation canvas become extremely clotted and complex with excessive consumption of screen space. This will result in lot of scrolling vertically and horizontally, in order to trace the required region of the ontology [6].

\subsubsection{D vs $2 \mathrm{D}$.}

$3 \mathrm{D}$ is not a good solution for ontology visualization requirements, since it causes stress escalation and cognitive overload to the users [6]-[8].

\subsubsection{Learning curve $\&$ information loss}

Use of techniques such as "Eular diagraming" results in information loss and additional comprehension barriers. Moreover, there is an integrated learning curve associated with the "Eular concepts" [9]-[11].

\subsection{Existing visualisation methods}

\subsubsection{Graph-based methods}

This is the most popular technique associated with ontology visualisation. Most of the users are familiar with this technique. The drawback is such that, when the ontology becomes complex, clutter and occlusion will occur as depicted in Figure 1. Henceforth with lots of nodes and edges crossing each other, this will make the presentation canvas cognitively complex to comprehend. Refer table 1 provided in the appendix to recognise several of ontology visualisation plugins that use this method [6], [12]-[14]. 


\subsubsection{Layout based methods}

There are multiple sub-categories of layout based methods available for ontology visualisation. These categories are namely; force-directed, radial, inverted-radial, circular and tree-maps. Radial, inverted-radial, and circular layouts are criticised for their excessive space wastage, rotated text representation and loss of hierarchical structure, which cause additional cognitive overload on the user for the realizations [15], [16]. Similarly, force-directed method also causes information losses [15].

However, usage of tree-maps has been acclaimed due to its ability of effective utilisation of the visualisation space available, provision of traversal experience to the users via drill-down interaction and the effective rationale of layered information handling. Moreover, all these features contribute towards effective handling of "scale versus information problem" that was already discussed. Hence, the tree-map layout has been effectively used for the visualisation of the concepts of Gene Ontology as many users have commended, due to its capability of reducing the cognitive overload [6], [13], [15], [17]-[19].

Furthermore, tree-maps preserve the hierarchical organisation of the information schemata and make the comprehension easier even for the non-technical specialists facilitated via the traversal interaction experience [6], [13], [15], [17]-[19]. Multiple literatures [20]-[22] have depicted that, tree-maps visualisation techniques can be fine-tuned to address most of the aforementioned requirements, since these are identified as a layout-based visualisation mechanism with the screen space-filling capability. Therefore, each pixel in the visualisation of a tree-map contributes to the information representation [21] with another value-added feature of 'content-aware resizing '[20]. Therefore, researchers claim that, with the proper configuration of the treemaps, it will be a fully dedicated visualisation artifact for the end-users, assisting effective "ontology sensemaking" [20], [21]. Also, a variety of applications of tree-maps in domains such as stock-market analysis and genetics have justified their ability to provide abstract representations, overcoming the cognitive barriers associated with comprehensions [6]. Furthermore, researchers have justified that, it will take lesser time to comprehend a tree-map based conceptualisation, compared to those of other modes of visualisation since its layer-based dimensionality reduction is accomplished though cognitive walk-through facility [21], [23].

However, one of the limitations of the tree-map is that, the information fed into the tree-map visualisation artifact needs to be properly grouped in a fine-grained manner enforcing the domain constrained mappings existing in the conceptualization. Otherwise, none of the afore-mentioned advantages of the treemaps can be leveraged [6], [13], [15], [17]-[19]. Refer Table 1 in appendix for a list of layouts method-based plugins.

\subsubsection{Eular diagraming method}

In relation to the Euler diagram-based methods, researches have claimed that, it is a novel representation mechanism, where the majority of the user-bases might not be familiar, in contrast with other visualisation categories. Furthermore, it has been pointed out that, there is a tendency of information loss in representing data associated with certain domains (i.e., property associated information). As a remedy of overcoming this information loss, in addition to the Eular diagrams interpretations, mathematical representations are also needed. Additionally, it is being suggested, to boost the understanding of the representations, piercing theory will assist end-users, even more in accurate comprehension of axioms presented in Euler diagrams. Therefore, it is identified as technically complex and the learning curve to be comparatively high in learning Euler diagrams and piercing theories. Therefore, proper comprehension of the knowledge representations, is definitely going to be an additional overload to the end-user [9]-[11]. Refer Table 1 in appendix for a list of Eular-method based plugins.

\subsection{Reflection}

According to the afore-mentioned discussion, it is apparent that there are challenges to be resolved in order to escalate the visual sense making clarity of the ontologies. Among the methods reviewed, other than the tree-maps, all other methods have several critical deficiencies. Even in tree-maps there is a necessity to properly group and feed the information. Or else provision of the afore-mentioned advantages of the tree-maps will not be feasible. Therefore, it can be concluded that, tree-maps are a potential solution to represent ontological schemata in a comprehensible manner. However, there needs to be a specifically defined algorithm to organise the schematic mappings of the ontology without any information loss, before feeding to the treemap chart framework. Proposed algorithm will make tree-map generation for the ontological schemata fully automated and manual configurations free. The remaining section of the paper discusses, how the aforementioned objective is accomplished. 


\section{RESEARCH METHOD}

Design Science Research Methodology [25] is customised and used for this research. In literature it is stated that, Design Science Research Methodology is ideal for investigating human centered interventions [24]-[26]. Ontological sense making via visualisation is also a human-centered activity initiated by both domain specialists and ontologists. Hence, it is concluded that, Design Science Research Methodology will be a suitable choice for this research. Customised version of the Design Science Research Methodology, has been utilized in this research as elaborated in Figure 2.

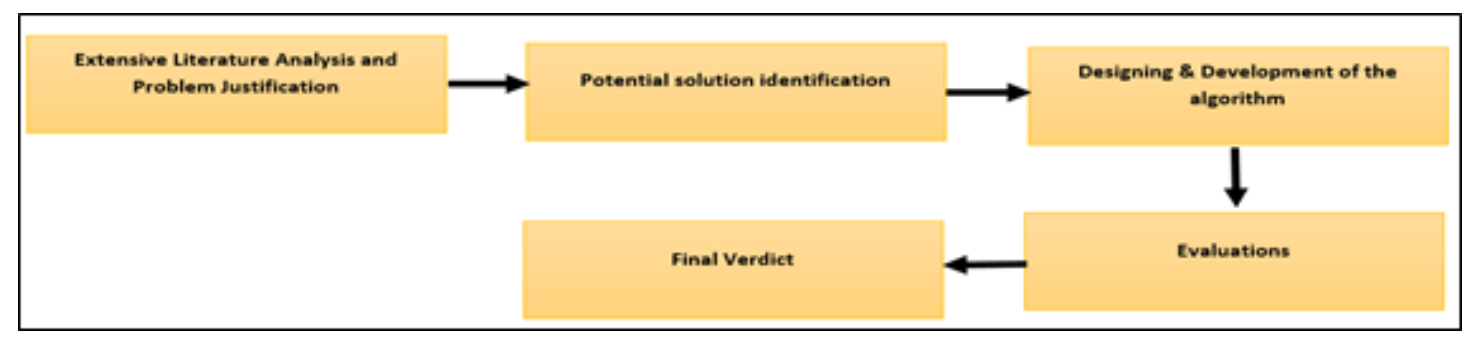

Figure 2. Customised version of the design science research methodology

As already depicted in Figure 2, the first step of the design science research methodology is to literary justify the problem of concern. This step has been already accomplished as per the contents discussed in the related work section. Second step is to derive on a potential solution. Again, as already conversed in the literature review, efficacy of the tree-maps are literary justified against the other alternatives. Hence, it`s decided to use tree-maps layout approach as the potential solution proposed for this research as well. In the third step, depending on the outcomes of the step one and two, an algorithm is designed to feed information to the tree-maps. Subsequently the designed algorithm is developed using java to exercise it on the real world use cases. The proposed algorithm is applied on three different domains to verify it's domain and schema independent capability. The steps followed in algorithm evaluation is methodically discussed in the evaluation section. Ultimately, iterative framework [34] is utilized to derive a final verdict about the algorithm`s capability to function in a domain and schema independent manner. Evaluation procedure utilized is elaborated in detailed in the evaluation section of this paper.

\section{RESULTS AND DISCUSSION}

High level communication flow of the proposed solution is represented in Figure 3. Flow of the algorithmic execution is illustrated in Figure 4. The first step of the algorithm is to extract classes, data properties, object properties, relationships with mappings preserved. This will assure, no mapping associated information in the ontology will be lost. Henceforth, all the extracted information sets are stored in a database as per the mappings residing in the ontology.

Once this step is completed, tuples can be extracted one-by-one according to the process illustrated in Figure 5. This process is iterative until all tuples are processed. Tuple associated information are passed to 'infocollectorTreemapRpt' method with a flag label claiming the type of the information sent to the method. This flag label acts to control conditional execution of the relevant code snippets associated with the appropriate conceptual aspects of the ontology (i.e., disjoint relationship), provided from the database tuple.

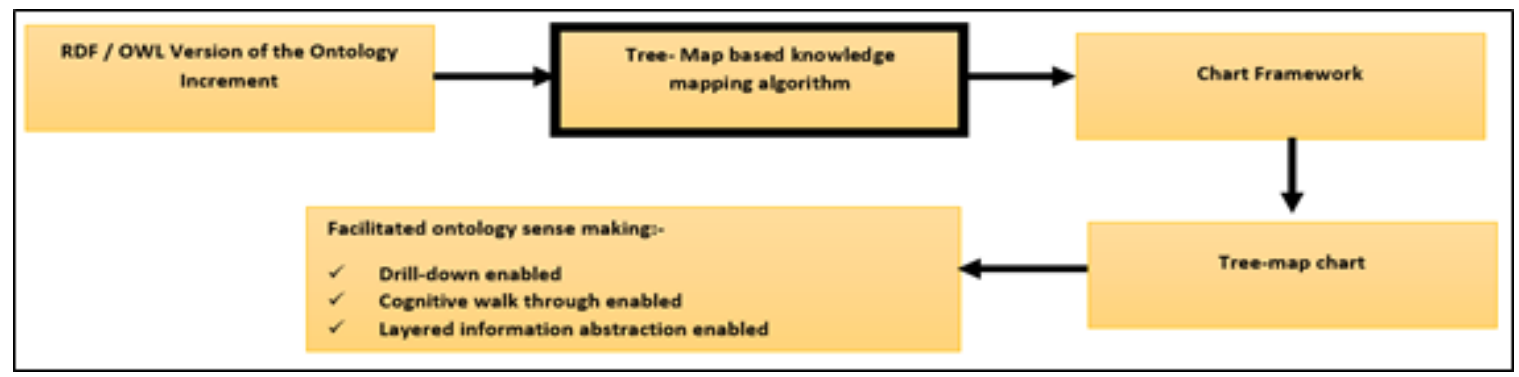

Comput. Sci. Inf. Technol., Vol. 2, No. 3, November 2021: 147 - 157 
Figure 3. High-level communication flow

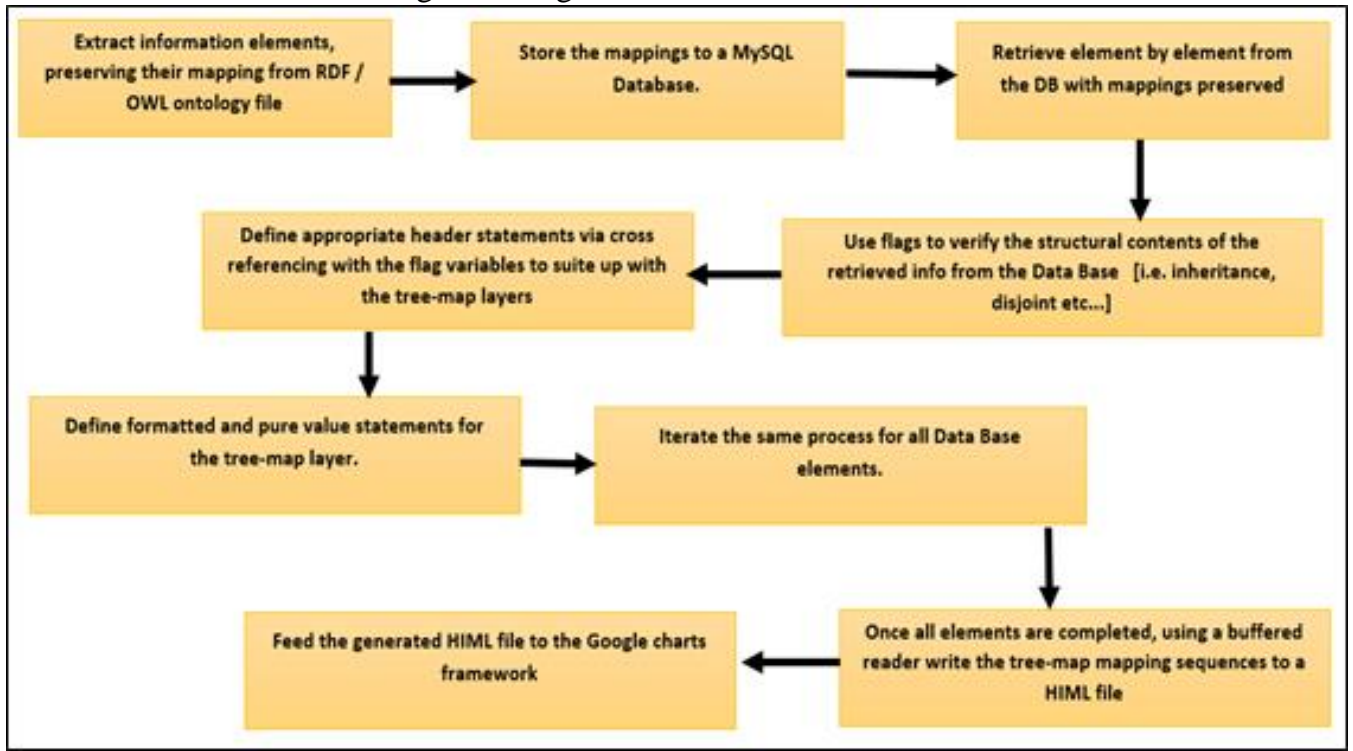

Figure 4. Algorithmic execution flow

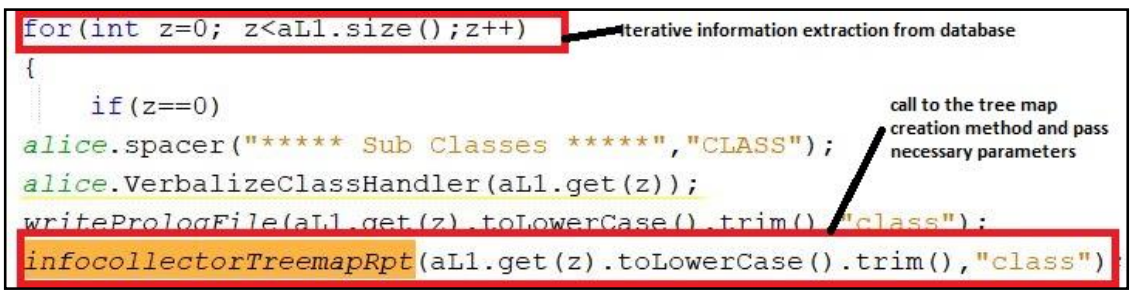

Figure 5. Tuple extraction logic

Inside conditional code snippets, first statement is for the definition of the header layout of the treemap. This will define one layer in the tree-map for the concept of concern (i.e. disjoint relationship). Additionally, level associated information is also needed to be stored in order to ensure smooth back-and-forth traversal, facilitating the cognitive walkthrough. This entire process is elaborated in Figure 6.

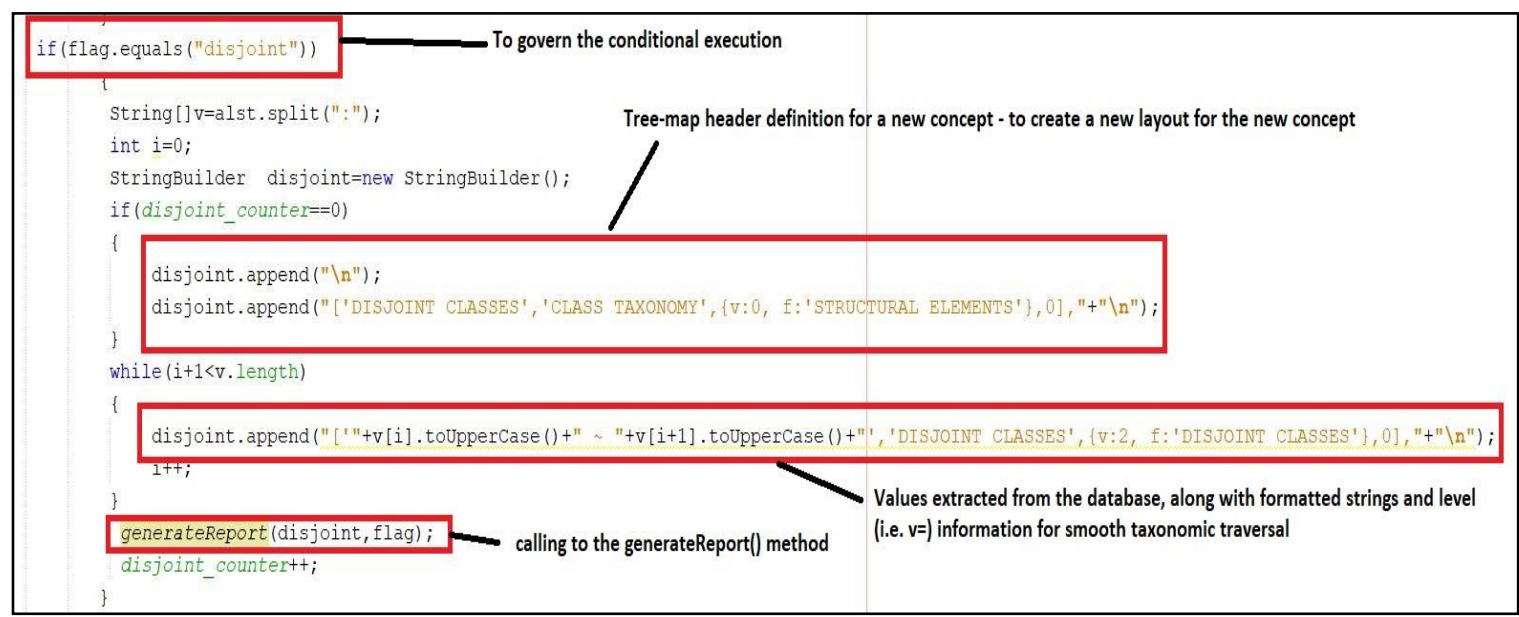

Figure 6. Tree-map layer organization logic 
Once all information of the ontology file is packaged and organised as in Figure 6, that information will be passed into the 'generateReport 'method. This method is responsible for the creation of the HTML version of the organised contents. This entire process is illustrated in Figure 7 code snippet. After examining on multiple charts frameworks, finally, Google charts framework is selected for the purpose of this research (i.e. charts.js framework doesn`t have tree-maps and D3 charts framework's syntax and configurations are very complex compared to the Google charts framework). Google chart's java script logic is separately maintained as an independent html file. Database contents are iteratively appended to another html file (i.e. Figure 7). Eventually, both these html files are merged together to derive the final functioning tree-map layout as depicted in Figure 8.

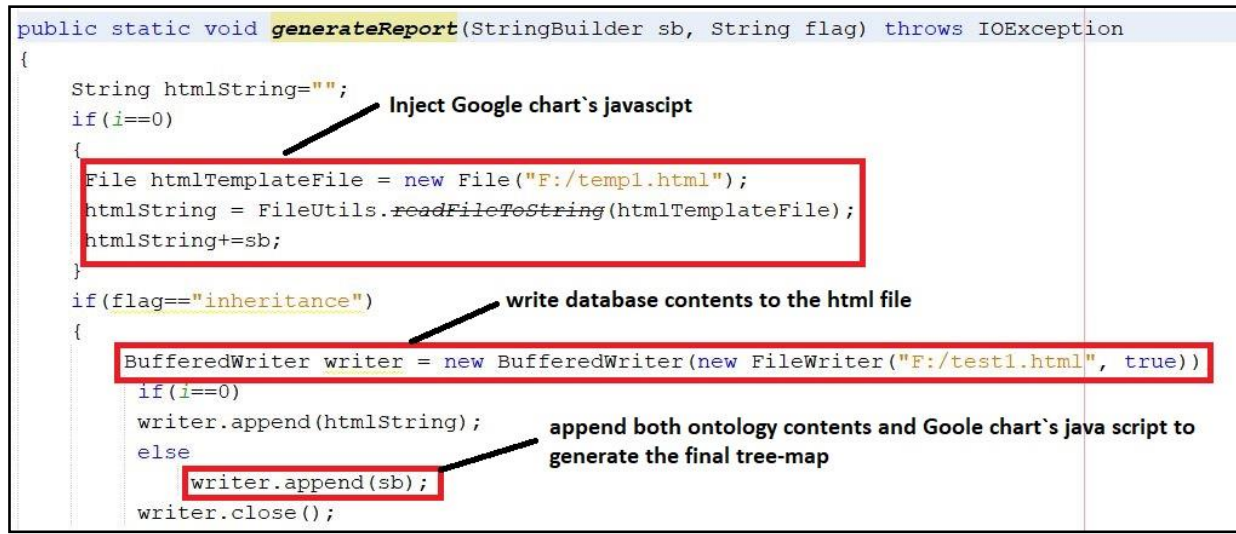

Figure 7. HTML version of the Tree-map file

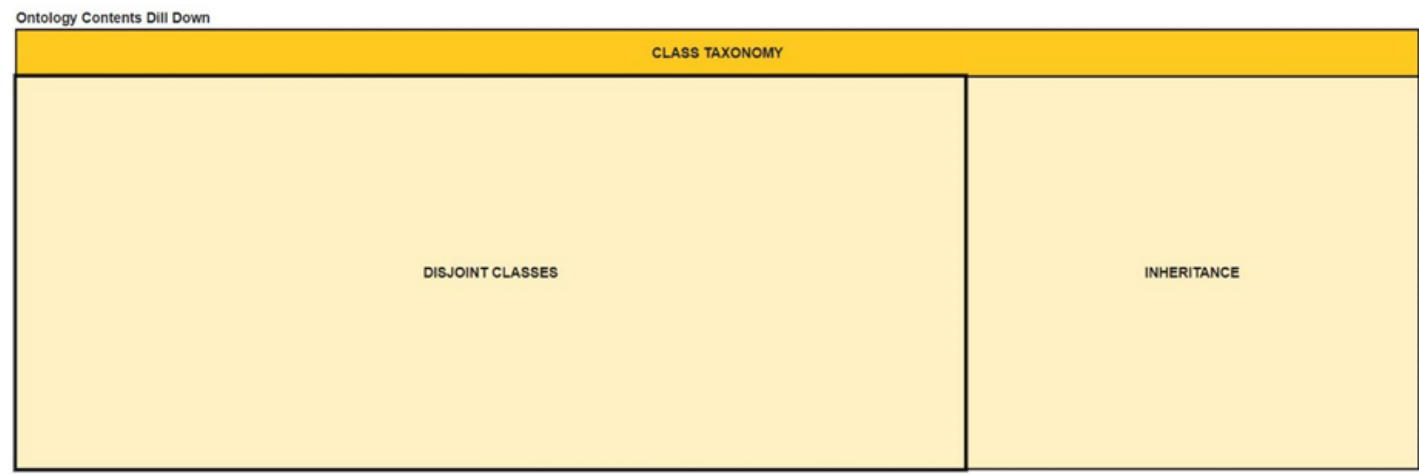

(a)

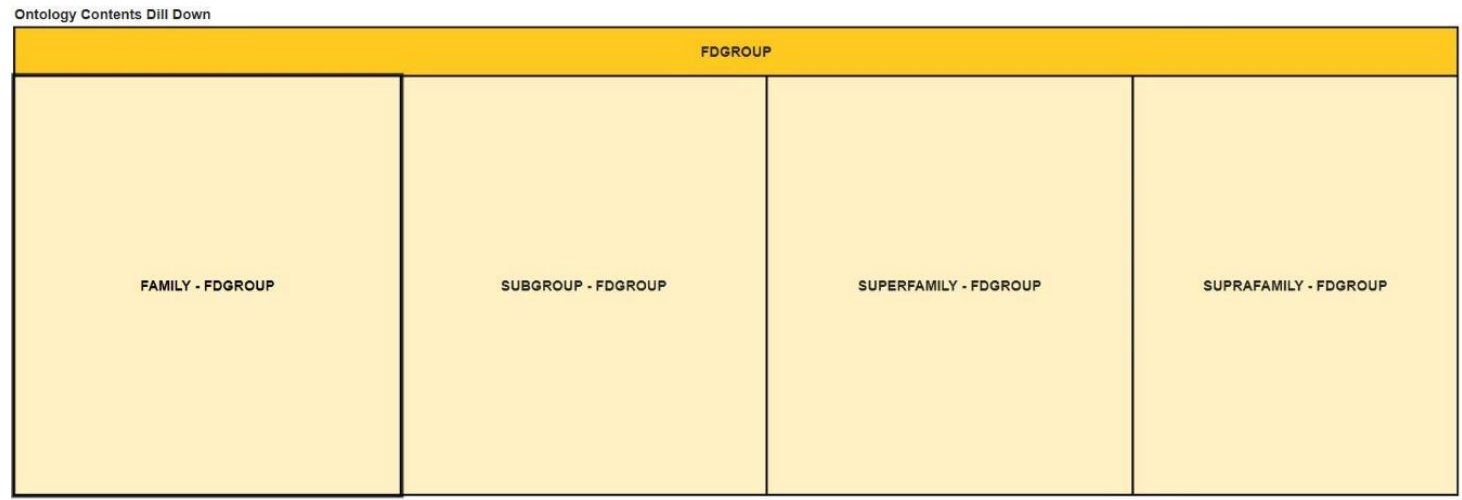

(b)

Figure 8. These figures are; (a) Functioning tree-map-at high level, (b) Functioning tree-map-drill down enabled 


\section{EVALUATION}

For the evaluation process six domain experts and three ontologists were independently selected. These domain specialists are from psychotherapy domain (two consultant psychiatrists), labor law domain (two lawyers) and marine biology domain (two biologists) respectively. All these six members were involved in ongoing ontology creation projects as domain specialists. Hence, they are equipped with an extensive knowledge about the taxonomy and schematic organisation of those respective three ontology increments, belonging into afore-mentioned three domains. As the first step, all of them were educated about this prototype developed and its applications via a workshop. Henceforth, they were requested to use this prototype in their latest ontology increments and comment on the opinions about the output as correct or incorrect.

All correct instances are classified as true positives and wrong instances (i.e. situations where taxonomic structure was not depicted accurately) as false positives. Henceforth, those values are used to calculate precision, recall and F-measure accuracy matrices. Domain specialists were informed to decide their opinions and $\log$ them as true positives or false positives. They were given a time duration of one week to complete this activity. By the end of the week, they were provided with a questionnaire and a specialised grid to rate their genuine experience with the prototype. The grid structure provided for capturing the ratings are depicted in Figure 9.

\begin{tabular}{|l|l|l|l|l|l|l|l|l|}
\hline 10 & 20 & 30 & 40 & 50 & 60 & 70 & 80 & 90 \\
\hline $\begin{array}{l}\text { Very } \\
\text { Poor }\end{array}$ & $\begin{array}{l}\text { Fairly OK, but major flaws } \\
\text { visible }\end{array}$ & $\begin{array}{l}\text { Good and acceptable - Only few minor } \\
\text { revisions }\end{array}$ & Exceptional \\
\hline
\end{tabular}

Figure 9. Rating grid

Questionnaire provided to the domain specialists contained questions on functionality of the prototype, accuracy of the prototype and the effectiveness of the proposed concept. The quantitative opinions provided by them and the accuracy matrices calculated are tabulated in Table 1 and Table 2 .

Table 1. Quantitative matrices on domain specialists' opinion

\begin{tabular}{llll}
\hline Domain Specialist-Domain of interest & Functionality & Accuracy & Effectiveness \\
\hline A - Psychotherapy & $80 \%$ & $80 \%$ & $70 \%$ \\
B- Psychotherapy & $70 \%$ & $80 \%$ & $80 \%$ \\
D- Law & $70 \%$ & $80 \%$ & $80 \%$ \\
E- Law & $80 \%$ & $90 \%$ & $70 \%$ \\
F- Marine Biology & $90 \%$ & $80 \%$ & $70 \%$ \\
G-Marine Biology & $80 \%$ & $80 \%$ & $80 \%$ \\
AVG & $78 \%$ & $82 \%$ & $75 \%$ \\
\hline
\end{tabular}

Table 2. Averaged Accuracy matrices

\begin{tabular}{ccc}
\hline \multicolumn{3}{c}{ Accuracy Matrices - Consolidated Results } \\
Precision & Recall & F-Measure \\
\hline 0.95 & 0.92 & 0.93 \\
\hline
\end{tabular}

Without discontinuing from the quantitative assessment, all the domain specialists were compelled to take part in a controlled interview session. The output of the controlled interview sessions conducted was summarised and tabulated in Table 3 (salient points extracted via doing a thematic assessment).

Table 3. Qualitative reflections of domain specialists

\begin{tabular}{lrl}
\hline Parameter & Viewpoints \\
\hline Functionality - overall 78\% & 1. & Useful for knowledge blending \\
& 2. & Drill down facilitates understanding \\
Accuracy - overall 83\% & 3. & Can traverse across the ontology \\
& 1. & Dimensionality Reduction and easy comprehension \\
& 2. & Simplicity and layered abstraction \\
Effectiveness - overall 77\% & 3. & Quick \& accurate \\
& 1. & Good to develop collaboration aspects as needed in ontology construction. \\
& 2. & No need of technical configurations \\
& 3. & Domain and schema independent.
\end{tabular}


In the second phase of the experiment, three ontologists were invited. They were briefed regarding the technical inner workings of the prototype while the results derived from the domain specialists' experiments were also exposed to them. Henceforth, they were also given the same grid to rate their opinions about the proposed prototype. However, their questionnaire contained questions on effectiveness of the prototype, novelty of the prototype and the architecture of the prototype. After collecting their quantitative assessment, they were also compelled to take part in a controlled interview session for deriving their qualitative reflections. The information collected are tabulated in Table 4 and Table 5.

Table 4. Quantitative matrices on ontologists opinion

\begin{tabular}{llll}
\hline Ontologist & Effectiveness & Novelty & Architecture \\
\hline A & $80 \%$ & $90 \%$ & $90 \%$ \\
B & $70 \%$ & $90 \%$ & $80 \%$ \\
C & $80 \%$ & $80 \%$ & $80 \%$ \\
AVG & $77 \%$ & $87 \%$ & $83 \%$ \\
\hline
\end{tabular}

Table 5. Qualitative reflections of ontologists

\begin{tabular}{ll}
\hline Parameter & Viewpoints \\
\hline Effectiveness-overall $77 \%$ & 1. Layered abstraction of information \\
& 2. Good solution for scale vs information density \\
& 3. Less susceptible for occlusion \\
4. Drill down enable cognitive walk-through & 1. schema independent \\
2. Domain independent & 3. No datasets needed for model training \\
& 4. Works with any domain \\
Architecture-overall $83 \%$ & 1. Cognitive walkthrough \\
& 2. Algorithm links the Google charts framework with the ontology \\
& 3. No need for configurations \\
& 4. Schema agnostic operation \\
5. Domain agnostic operation
\end{tabular}

Eventually iterative framework was utilised to assess the objective accomplishment of the entire research (i.e. independent ontologists was invited for this task). Iterative framework is an established framework for opinion mining [34]. In this research, it is used to assess the accomplishment of the research objective of this research. These details are tabulated in Table 6. Entire evaluation process is depicted in Figure 10.

Table 6. Iterative framework`s results

\begin{tabular}{|c|c|}
\hline Iterative Framework Step & Justification Elaborations \\
\hline \multirow[t]{2}{*}{$\begin{array}{l}01 \text { - What are the data telling } \\
\text { me? }\end{array}$} & $\begin{array}{l}\text { In quantitative experiment conducted, domain specialists have given a } 78 \% \text { of averaged consent for } \\
\text { the proposed resolution. }\end{array}$ \\
\hline & $\begin{array}{l}\text { Additionally, the qualitative feedback provided (i.e. summary is documented in tables } 3 \text { and 5) by } \\
\text { both domain specialist and ontologists also depicts positive attributes about the entire framework, } \\
\text { and it`s workarounds. }\end{array}$ \\
\hline 02- What do I want to know? & $\begin{array}{l}\text { How effective is the proposed visualization resolution in accomplishing the stipulated research } \\
\text { objective? }\end{array}$ \\
\hline 03 - Is there a dialectical & Yes. \\
\hline $\begin{array}{l}\text { relationship in step } 01 \text { and step } \\
02 \text { ? }\end{array}$ & $\begin{array}{l}\text { Both qualitative and quantitative results yielded has depicted the efficacy of the proposed } \\
\text { resolution, in terms of the research objective to be addressed. }\end{array}$ \\
\hline
\end{tabular}




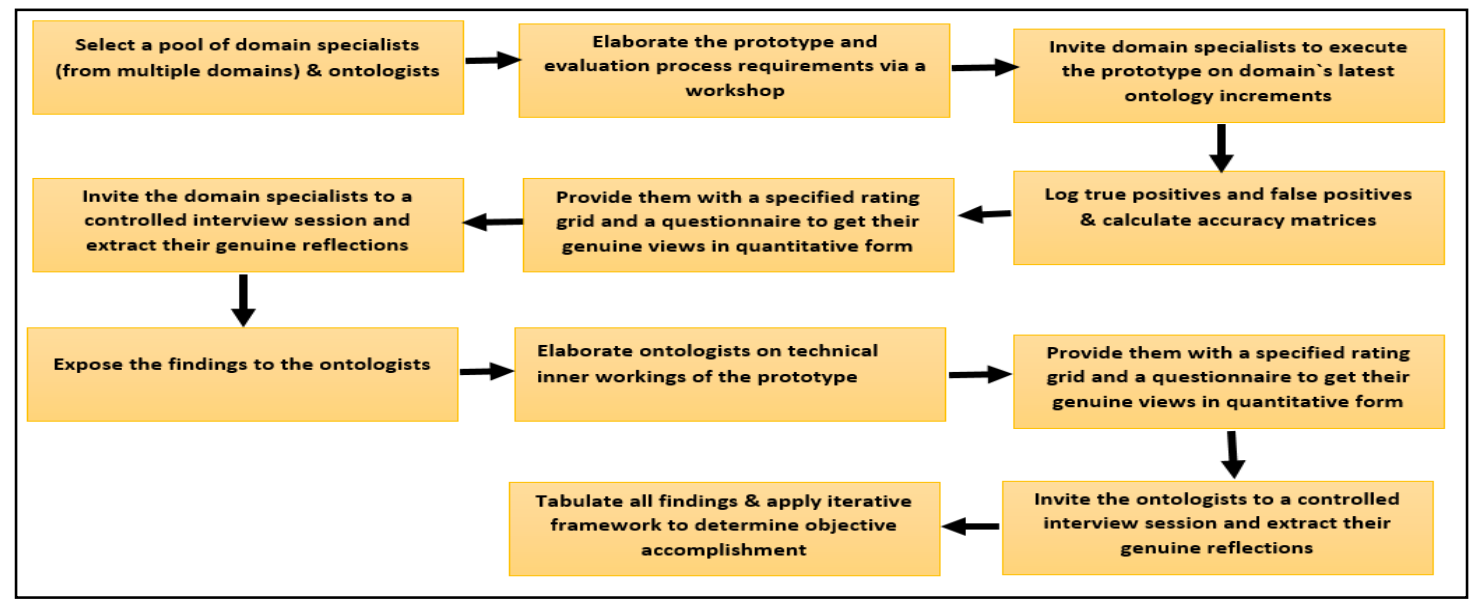

Figure 10. Entire evaluation flow

\section{CONCLUSION}

It was already conversed that there is a requirement for a sensible solution for effective ontology sense making. Existing methods had numerous challenges as already discussed in the section for related work. Among all existing approaches, tree-maps seem to provide a sensible resolution to this problem. However, in order to gain the real advantage from the tree-map, information extracted from the ontology needs to be fed to the tree-map, preserving the taxonomic mappings and schematic information in the ontology. The objective of this research is to propose an algorithm to accomplish that shortcoming. Proposed algorithm is capable of extracting all required information from the ontology file and methodically feeding it to the tree-map without loss of information (refer Tables 1-5 as justifications). Therefore, this has resulted in effective tree-map operation for ontology sense making, as already justified through the evaluation outcomes as well (refer Tables 1-5). As for future recommendations, influence will be given to further enhancement of the human computer interaction aspect of the proposed tool.

\section{APPENDIX}

Appendix: Visualization Plugin Comparison Appendix - Table-1 Visual Plugin Comparison

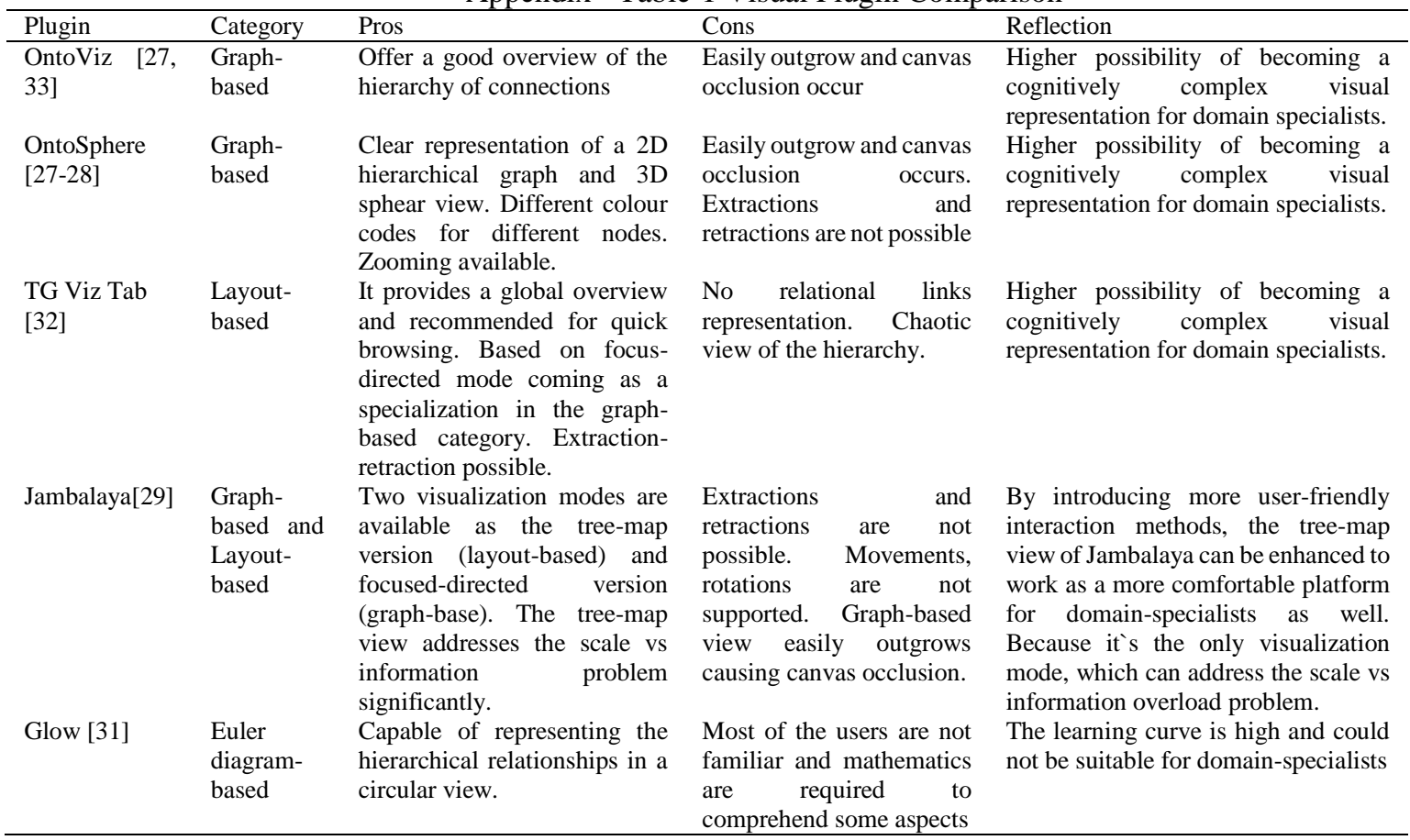


Appendix: Visualization Plugin Comparison (Continued)

Appendix - Table-1 Visual Plugin Comparison (Continued)

\begin{tabular}{|c|c|c|c|c|}
\hline Plugin & Category & Pros & Cons & Reflection \\
\hline $\begin{array}{l}\text { Swoop } \\
{[30]}\end{array}$ & $\begin{array}{l}\text { Euler } \\
\text { diagram- } \\
\text { based }\end{array}$ & $\begin{array}{l}\text { Capable of representing the } \\
\text { hierarchical relationships in a circular } \\
\text { view. Disjointness also can be } \\
\text { presented via non-overlapping of } \\
\text { circles }\end{array}$ & $\begin{array}{l}\text { Cardinality and property information } \\
\text { can be lost. Easily outgrow and canvas } \\
\text { and mathematics are required to } \\
\text { comprehend some aspects }\end{array}$ & $\begin{array}{l}\text { The learning curve is } \\
\text { high and could not be } \\
\text { suitable for domain- } \\
\text { specialists }\end{array}$ \\
\hline
\end{tabular}

\section{REFERENCES}

[1] L. Merino, E. Kozlova, O. Nierstrasz and D. Weiskopf, "VISON: An Ontology-Based Approach for Software Visualization Tool Discoverability," 2019 Working Conference on Software Visualization (VISSOFT), Cleveland, OH, USA, 2019, pp. 45-55, doi: 10.1109/VISSOFT.2019.00014.

[2] Park, S., Kim, S., \& Ha, Y. "Scalable visualization for DBpedia ontology analysis using Hadoop." Software: Practice and Experience, vol. 45, no. 8, 1103-1114, 2015. https://doi.org/10.1002/spe.2310

[3] S. Carpendale et al., "Ontologies in Biological Data Visualization," in IEEE Computer Graphics and Applications, vol. 34, no. 2, pp. 8-15, Mar.-Apr. 2014, doi: 10.1109/MCG.2014.33.

[4] Anikin, A., Litovkin, D., Kultsova, M., Sarkisova, E., \& Petrova, T. Ontology Visualization: Approaches and Software Tools for Visual Representation of Large Ontologies in Learning. Communications in Computer and Information Science, pp. 133-149, 2017. doi:10.1007/978-3-319-65551-2_10

[5] Motta, E., Mulholland, P., Peroni, S., d'Aquin, M., Gomez-Perez, J.M., Mendez, V., Zablith, F.: A novel approach to visualizing and navigating ontologies. In: Aroyo, L., Welty, C., Alani, H., Taylor, J., Bernstein, A., Kagal, L., Noy, N., Blomqvist, E. (eds.) The Semantic Web \{ ISWC 2011: 10th International Semantic Web Conference, Bonn, Germany, October 23-27, 2011

[6] Silva, I. C., Santucci, G., \& Freitas, C. M. Visualization and analysis of schema and instances of ontologies for improving user tasks and knowledge discovery. Journal of Computer Languages, vol. 51, pp. 28-47, 2019. doi:10.1016/j.cola.2019.01.004

[7] Saghafi, A. Visualizing ontologies - a literature survey, Graph-based representation and reasoning: 22nd International Conference on Conceptual Structures, ICCS 2016, Annecy, France, July 5-7, 2016, Proceedings, Cham: Springer International Publishing, pp. 204-221, 2016.

[8] Fiedler, Rilling, Bogen, \& Herder. "Multimodal Interaction Techniques in Scientific Data Visualization - An Analytical Survey." Proceedings of the 10th International Conference on Computer Graphics Theory and Applications. 2015. doi:10.5220/0005296404310437

[9] Stapleton, G., Chapman, P., Rodgers, P., Touloumis, A., Blake, A., \& Delaney, A. "The efficacy of Euler diagrams and linear diagrams for visualizing set cardinality using proportions and numbers." PLOS ONE, vol. 14, no. 3, p. e0211234, 2019. doi:10.1371/journal.pone.0211234

[10] G. Stapleton, L. Zhang, J. Howse and P. Rodgers, "Drawing Euler Diagrams with Circles: The Theory of Piercings," in IEEE Transactions on Visualization and Computer Graphics, vol. 17, no. 7, pp. 1020-1032, July 2011, doi: 10.1109/TVCG.2010.119.

[11] P. Rodgers, G. Stapleton, J. Flower and J. Howse, "Drawing Area-Proportional Euler Diagrams Representing Up To Three Sets," in IEEE Transactions on Visualization and Computer Graphics, vol. 20, no. 1, pp. 1-1, Jan. 2014, doi: 10.1109/TVCG.2013.104.

[12] Gawich, M., Alfonse, M., Aref, M., \& Salem, A. M. "Ontology Maintenance System for Rheumatoid Disease." Procedia Computer Science, vol. 154, pp. 341-346, 2019. doi:10.1016/j.procs.2019.06.049

[13] Nazemi, K., Burkhardt, D., Ginters, E., \& Kohlhammer, J. "Semantics Visualization - Definition, Approaches and Challenges.” Procedia Computer Science, vol. 75, pp. 75-83, 2015. doi:10.1016/j.procs.2015.12.216

[14] Öztürk, Ö., \& Açikgöz, H. G. "Onyx: A new Canvas-based tool for visualizing biomedical and health ontologies." Expert Systems, vol. 37, no. 5, pp. e12380, 2019. doi:10.1111/exsy.12380

[15] Dudáš, M., Lohmann, S., Svátek, V., \& Pavlov, D. "Ontology visualization methods and tools: a survey of the state of the art.” The Knowledge Engineering Review, vol. 33, pp. 1-39, 2018. doi:10.1017/s0269888918000073

[16] Lohmann, S., Negru, S., Haag, F., \& Ertl, T. (2016). Visualizing ontologies with VOWL. Semantic Web, 7(4), 399419. doi:10.3233/sw-150200

[17] Hahn. (2014). Visualization of Varying Hierarchies by Stable Layout of Voronoi Treemaps. Proceedings of the 5th International Conference on Information Visualization Theory and Applications. doi:10.5220/0004686200500058

[18] Baehrecke, Dang, Babaria, \& Shneiderman. "Visualization and analysis of microarray and gene ontology data with treemaps.” BMC Bioinformatics., vol. 5, no. 84, pp. 1-12, 2004.

[19] B. Fu, N. Noy, M. Storey, "Eye tracking the user experience - an evaluation of ontology visualization techniques," Semantic Web - Interoperability, Usability, Applicability, vol. 8, no. 1, pp. 23-41, 2017.

[20] Li, C., Baciu, G., Wang, Y., \& Zhang, X. "Fast content-aware resizing of multi-layer information visualization via adaptive triangulation." J. Vis. Lang. Comput., vol. 45, pp. 61-73, 2017.

[21] Yalçin, M.A., Elmqvist, N., \& Bederson, B.B. "Raising the Bars: Evaluating Treemaps vs. Wrapped Bars for Dense Visualization of Sorted Numeric Data. Graphics Interface." GI '17: Proceedings of the 43rd Graphics Interface Conference, pp. 41-49, January 2017. 
[22] Wiens, V., Lohmann, S., \& Auer, S. "Semantic Zooming for Ontology Graph Visualizations." Proceedings of the Knowledge Capture Conference on - K-CAP 2017. 2017, doi:10.1145/3148011.3148015

[23] Herr, D., Han, Q., Lohmann, S., \& Ertl, T. (2016). "Visual Clutter Reduction through Hierarchy-based Projection of High-dimensional Labeled Data. Graphics Interface." Proceedings of Graphics Interface 2016: Victoria, British Columbia, Canada, pp. 109-116, 1-3 June 2016.

[24] Hybs, "Beyond the Interface: A Phenomenological View of Computer Systems Design," Leonardo, vol. 29, no. 3, p. 215,1996

[25] McCarthy, J. "Circumscription-A Form of Non-Monotonic Reasoning." Artificial Intelligence, vol. 13, no. 1-2, pp. 27-39, 1980.

[26] Sarkar and Cybulski, "Evaluation of Phenomenological Findings in IS Research: A Study in Developing WebBased IS," European Conference on Information Systems, rep., 2004.

[27] Sivakumar, Arivoli. “Ontology Visualization Protégé Tools - a Review.” International Journal of Advanced Information Technology, vol. 1, no. 4, pp. 1-11, August 2011

[28] Musen, M. A. "The protégé project." AI Matters, vol. 1, no. 4, pp. 4-12, 2015. doi:10.1145/2757001.2757003

[29] Musen, M. A., \& The Protégé Team. (2013). Protégé Ontology Editor. Encyclopedia of Systems Biology, 1763-1765. doi:10.1007/978-1-4419-9863-7_1104

[30] Kalyanpur, A., Parsia, B., Sirin, E., Grau, B.C., \& Hendler, J.A. “Swoop: A Web Ontology Editing Browser.” J. Web Semant., vol. 4, no. 2, pp. 144-153, 2006.

[31] Hop, W., Ridder, S.D., Frasincar, F., \& Hogenboom, F. "Using Hierarchical Edge Bundles to visualize complex ontologies in GLOW." SAC '12: Proceedings of the 27th Annual ACM Symposium on Applied Computing, pp. 304 311, 2012. https://doi.org/10.1145/2245276.2245338.

[32] Malik, S.K., \& Rizvi, S.A. "A group housing society ontology in Swoop 2.3 Beta 4 and Protege 3.4.4.” International Journal of Autonomic Computing, vol. 2, no. 1, pp. 21-38. 2014

[33] Sintek, M. Ontoviz tab: Visualizing protege ontologies, 2003.

[34] Srivastava, P., \&amp; Hopwood, N. "A Practical Iterative Framework forQualitative Data Analysis." International Journal of Qualitative Methods, vol. 8, no. 1, pp. 76-84, 2009.

\section{BIOGRAPHIES OF AUTHORS}
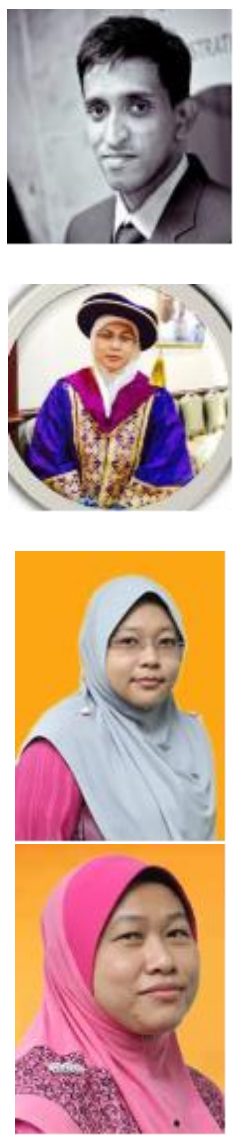

Kaneeka Vidanage -Kaneeka is postgraduate level researcher and a $\mathrm{PhD}$ research student attached to University Malaysia Terengganu. Kaneeka holds, his bachelors in Managing Information Systems from National University of Ireland, Master of Computer Science from, University of Colombo and Master of Philosophy (M.Phil) in Semantic Web and ontology based intelligence from University of Colombo.

Noor Maizura Mohamad Noor - Noor Maizura is a professor in computer science, attached to University Malaysia Terengganu. Professor, Maizura got in PhD from Manchester University UK, specializing in the area of decision sciences.

Rosmayati Mohemad -Rosmayati Mohemad is a doctor in computer science, attached to University Malaysia Terengganu. Doctor, Rosmayati got in PhD from National University of Malaysia, specializing in the area of semantic web and ontologies

Zuriana Abu bakar -Zuriana Abu barkar is a doctor in computer science, attached to University Malaysia Terengganu. Doctor, Zuriana got in PhD from Queensland University, Australia, speializing in the area of Human Computer Interaction 